\title{
CONFORMAL CONNECTIONS AND CONFORMAL TRANSFORMATIONS
}

\author{
BY \\ NOBORU TANAKA
}

Introduction. In the previous paper [5], we have studied the groups of projective transformations of affinely connected manifolds by the application of the theory of normal projective connections. The main purpose of the present paper is to study the conformal properties of complete Riemannian spaces of some special type by the application of the theory of normal conformal connections.

We shall introduce a family of Riemannian spaces, the elements of which are characterized by conditions on the Ricci tensor fields and will be called of type $\mathfrak{S}$ (Definition 4 ). Einstein spaces are of type $\mathfrak{S}$ and, in general, a Riemannian space of type $\subseteq$ is locally isomorphic with the direct product of two Einstein spaces of different signs. Our main result (Theorem 1) states that two conformally equivalent Riemannian metrics on a manifold which are complete and of type $\mathfrak{S}$ necessarily coincide, except the case where either of the two metrics is an Einstein metric with the vanishing or negative Ricci tensor field. Some results are also obtained in the exceptional case. We see from this theorem that the group of all conformal transformations of a complete Riemannian space of type $\subseteq$ coincides with the group of all isometries, except the case of an Einstein space with the vanishing or negative Ricci tensor field (Theorem 2).

The proof of Theorem 1 is based on the theory of normal conformal connections; it has been suggested by a result of K. Yano and S. Sasaki [8] stating that the conformal holonomy group of a Riemannian space of type ऽ fixes a point or a sphere. In $\S \S 1-4$, we shall show how to construct the normal conformal connection when an arbitrary class of conformally equivalent Riemannian metrics is given. The conditions for the normal conformal connection are exhibited in Proposition 5.

Finally we mention that our formulation of the normal conformal connection is efficacious for many other problems concerning the group of conformal transformations or the conformal holonomy group, for example, for such a problem as is treated in [8], cited above. We want to take up these problems at another occasion.

I would like to express my sincere thanks to Professor K. Nomizu for his constant encouragement and kind interest in my work.

Remarks about notations and terminologies. Throughout this paper, we follow, in principle, the notations and terminologies adopted in the author's

Received by the editors January 2, 1958. 
paper [5]. By a manifold (a mapping) we shall always mean that of class $C^{\infty}$.

Let $M$ be a manifold. We shall denote by $M_{p}$ the tangent vector space to $M$ at a point $p$ of $M$ and by $\mathfrak{X}(M)$ the set of all vector fields on $M$. $\mathfrak{X}(M)$ may be considered as a vector space over the field of all real numbers.

Let $M$ and $N$ be two manifolds and let $f$ be a mapping of $M$ into $N$. For each tangent vector $X$ to $M, f X$ denotes the result of applying the differential of $f$ to $X$ (in Chevalley's notation, $f X=d f(X)$ ). Now, assume that $N$ is a Lie group. For each $p \in M$ and $X \in M_{p}$, we denote by $f^{-1} d f(X)$ the result of applying the differential of the mapping $M \ni q \rightarrow f^{-1}(p) f(q) \in N$ to $X$. Then, $f^{-1} d f(X)$ is naturally identified with an element of the Lie algebra $\mathfrak{n}$ of $N$ and the mapping $X \rightarrow f^{-1} d f(X)$ defines an $\mathfrak{n}$-valued 1 -form on $M$, which we denote by $f^{-1} d f$.

Let $N$ be a manifold and let $u(t)$ be a curve in $M$. We denote by $u^{\prime}(t)$ the tangent vector to $u(t)$ at $t$. In the case where $N$ is a Lie group, we denote by $u(t)^{-1} u^{\prime}(t)$ the result of applying the differential of the left translation $\sigma$ $\rightarrow u(t)^{-1} \sigma$ to $u^{\prime}(t)$. If we set $X(t)=u(t)^{-1} u^{\prime}(t)$, then $X(t)$ may be considered as a curve in the Lie algebra $\mathfrak{n}$ of $N$. Conversely, for each curve $X(t)$ in $\mathfrak{n}$ there exists one and only one curve $u(t)$ in $N$ such that $u(t)^{-1} u^{\prime}(t)=X(t)$ and $u(0)=e$ (the unit element of $N$ ) [6, p. 29]. In $\S \S 5$ and 6, we make use of this fact.

Let $P(M, G)$ be a principal fiber bundle over a base space $M$ with structure group $G$. For each $\sigma \in G$, we denote by $R_{\sigma}$ the right translation of $P$ onto itself which corresponds to $\sigma$. For each $z \in P, G_{z}$ denotes the subspace of $P_{z}$ which is tangent to the fiber through $z$. Let $\mathfrak{g}$ be the Lie algebra of $G$. For each $A \in \mathfrak{g}, A^{*}$ denotes the vector field on $P$ induced by the one-parameter group $R_{a(t)}$, where $a(t)=\exp t A$. Then, $G_{z}$ is equal to the subspace of $P_{z}$ composed of all the elements $A_{z}^{*}$ where $A$ runs over g.

Let $P(M, G)$ and $P^{\prime}\left(M, G^{\prime}\right)$ be two principal fiber bundles over the same base space $M$. Let $f$ be a homomorphism of $G$ into $G^{\prime}$. A mapping $f$ of $P$ into $P^{\prime}$ is called a homomorphism corresponding to the homomorphism $f$ of $G$ into $G^{\prime}$, if, for each $z \in P$ and $\sigma \in G, f(z \cdot \sigma)=f(z) \cdot f(\sigma)$ and if it induces the identity transformation of $M$ onto itself. In the case where $G$ is a subgroup of $G^{\prime}$, a homomorphism $f$ of $P$ into $P^{\prime}$ corresponding to the injection of $G$ into $G^{\prime}$ is called an injection. If we identify $P$ with a submanifold of $P^{\prime}$ by the injection, we shall say that $P$ is a subbundle of $P^{\prime}$ or $P$ is contained in $P^{\prime}$.

1. Möbius space. In this section, we define the Möbius space as a homogeneous space and study the homogeneous structure from an infinitesimal point of view.

(1.1) Let $F_{n+2}$ be an $(n+2)$-dimensional vector space over the field $R$ of all real numbers. We consider a fixed decomposition of $F_{n+2}$ :

$$
F_{n+2}=F_{0}+F_{n}+F_{\infty},
$$

where $F_{0}$ and $F_{\infty}$ are 1-dimensional subspaces of $F_{n+2}$ and $F_{n}$ is an $n$-dimensional subspace of $F_{n+2}$. Choose, once for all, bases $\xi_{0}$ and $\xi_{\infty}$ in $F_{0}$ and $F_{\infty}$ 
respectively. Denote by $F_{n}^{*}$ the dual space of $F_{n}$ and by $\langle\xi, E\rangle$ the product between $\xi \in F_{n}$ and $E \in F_{n}^{*}$.

(1.2) We consider an inner product $\phi(X, Y)$ in $F_{n+2}$ satisfying the following conditions:

(i) $\phi\left(\xi_{0}, \xi_{0}\right)=\phi\left(\xi_{\infty}, \xi_{\infty}\right)=\phi\left(\xi_{0}, \xi\right)=\phi\left(\xi_{\infty}, \xi\right)=0, \phi\left(\xi_{0}, \xi_{\infty}\right)=-1$ where $\xi \in F_{n}$;

(ii) The restriction of $\phi$ to $F_{n}$ is positive definite. We shall denote by $\phi$ the linear isomorphism of $F_{n}$ onto $F_{n}^{*}$ defined by $\left\langle\xi, \phi\left(\xi^{\prime}\right)\right\rangle=\phi\left(\xi, \xi^{\prime}\right)$. Let $P_{n+1}$ be the $(n+1)$-dimensional projective space constructed from $F_{n+2}$. We denote by $M_{n}$ the quadric in $P_{n+1}$ defined by the quadratic equation in $F_{n+2}: \phi(X, X)$ $=0$. We denote by $C$ the cone in $F_{n+2}-(0)$ defined by the same equation and by $\omega$ the projection of $C$ onto $M_{n}$. We set $o=\omega\left(\xi_{0}\right)$ and $\infty=\omega\left(\xi_{\infty}\right)$.

(1.3) Let $N$ be the subgroup of the general linear group of $F_{n+2}$ which leaves the inner product $\phi(X, Y)$ invariant and let $\omega$ be the projection of $N$ into the projective group of $P_{n+1}$. If we set $M(n)=\omega(N)$, we see that $M(n)$ leaves $M_{n}$ invariant; more precisely, $M(n)$ operates on $M_{n}$ as follows:

$$
\omega(\sigma) \omega(X)=\omega(\sigma X) \text { for all } \sigma \in N \text { and } X \in C .
$$

An important result is that $M(n)$, considered as a transformation group on $M_{n}$, is effective and transitive on $M_{n}$. Thus $M_{n}$ may be represented as a homogeneous space

$$
M_{n}=M(n) / M^{\prime}(n),
$$

where $M^{\prime}(n)$ denotes the isotropy group of $M(n)$ at $o$. We call this homogeneous space the $n$-dimensional Möbius space. It is homeomorphic with the $n$-dimensional sphere. $M(n)$ is called the Möbius group of dimension $n$.

(1.4) Let $O(n)$ be the orthogonal group of $F_{n}$ with respect to the inner product $\phi$ and let $S(n)$ be the similarity group of $F_{n}$. Let $R_{+}$be the multiplicative group of all positive real numbers. We know that $S(n)$ is the subgroup of the general linear group of $F_{n}$ composed of all the elements $\lambda \sigma$ where $\lambda \in R_{+}$and $\sigma \in O(n)$. If we identify $R_{+}$with a subgroup of $S(n)$ by the isomorphism $R_{+} \ni \lambda \rightarrow \lambda 1 \in S(n)$, where 1 denotes the unit element of $S(n)$, then we have the following expression

$$
S(n)=O(n) \cdot R_{+} \text {(direct product). }
$$

We denote by $s$ and $\rho$ the corresponding projections of $S(n)$ onto $O(n)$ and $R_{+}$ respectively.

(1.5) Now we construct a Lie group, denoted by $L\left(S(n), F_{n}^{*}\right)$, from $S(n)$ and $F_{n}^{*}$ as follows: The underlying manifold is equal to $S(n) \times F_{n}^{*}$ and the operation of multiplication is given by $(\tau, E) \cdot\left(\tau^{\prime}, E^{\prime}\right)=\left(\tau \tau^{\prime},{ }^{t} \tau^{\prime} E+E^{\prime}\right)$, where $\tau, \tau^{\prime} \in S(n)$ and $E, E^{\prime} \in F_{n}^{*}$. Namely, $L\left(S(n), F_{n}^{*}\right)$ is identical with the socalled abelian extension of $S(n)$ with respect to the star representation of $S(n)$. We show that $L\left(S(n), F_{n}^{*}\right)$ may be identified with $M^{\prime}(n)$. Indeed, we 
define an isomorphism $\psi$ of $L\left(S(n), F_{n}^{*}\right)$ into $N$ by $\psi(\sigma)=\tilde{\tau} \cdot \tilde{E}$ for all $\sigma=(\tau, E)$ $\in S(n) \times F_{n}^{*}$, where $\tilde{\tau}$ and $\tilde{E}$ denote the elements of $N$ defined respectively as follows:

$$
\begin{aligned}
& \tilde{\tau} \xi_{0}=\rho(\tau)^{-1} \xi_{0}, \tilde{\tau} \eta=s(\tau) \eta, \tilde{\tau} \xi_{\infty}=\rho(\tau) \xi_{\infty} \\
& \tilde{E} \xi_{0}=\xi_{0}, \tilde{E} \eta=\langle\eta, E\rangle \xi_{0}+\eta, \tilde{E} \xi_{\infty}=2^{-1}\left\langle\phi^{-1} E, E\right\rangle+\phi^{-1} E+\xi_{\infty},
\end{aligned}
$$

where $\eta \in F_{n}$. We see easily that $\omega \circ \psi$ gives an isomorphism of $L\left(S(n), F_{n}^{*}\right)$ with $M^{\prime}(n)$. In the following, we identify $S(n)$ with a subgroup of $M^{\prime}(n)$ by the isomorphism $S(n) \ni \tau \rightarrow(\tau, 0) \in M^{\prime}(n)$ and we write $\exp E=(1, E)$. With this notation, we see that every element $\sigma$ of $M^{\prime}(n)$ is expressed uniquely as

$$
\sigma=l(\sigma) \cdot \exp E(\sigma),
$$

where $l(\sigma)$ is in $S(n)$ and $E(\sigma)$ in $F_{n}^{*}$. The correspondence $\sigma \rightarrow l(\sigma)$ gives a homomorphism of $M^{\prime}(n)$ onto $S(n)$. Finally we have the following diagram:

$$
\begin{aligned}
& M(n) \stackrel{\text { inj. }}{M^{\prime}(n)} \stackrel{l}{S(n)}^{l} \\
& \text { inj. } s \text { inj. } \\
& O(n)
\end{aligned}
$$

where inj. means injection and where $s \circ l(\sigma)=\sigma$ for all $\sigma \in O(n)$.

(1.6) Let $8(n)$ be the Lie algebra of $S(n)$ which is identified with a subalgebra of the Lie algebra of all endomorphisms of $F_{n}$. Then, corresponding to the decomposition of $S(n)$ given in (1.4), we have the decomposition of $8(n)$ :

$$
8(n)=\mathfrak{o}(n)+R .
$$

For each $A \in \mathcal{B}(n)$, we denote by $A_{\mathrm{o}(n)}$ and $A_{R}$ the $\mathrm{o}(n)$ - and $R$-components of $A$ respectively.

(1.7) In what follows, we study the Lie algebra of $M(n)$. Let $\mathfrak{n}$ be the Lie algebra of $N$ which is identified with the Lie algebra composed of all the endomorphisms of $F_{n+2}$ which leave the inner product $\phi$ in $F_{n+2}$ invariant. First of all, $\mathfrak{n}$ may be identified with the Lie algebra of $M(n)$, because the kernel of $\omega: N \rightarrow M(n)$ is a discrete subgroup of $N$. Now consider the formal direct sum $\mathfrak{m}(n)$ of the three vector spaces $F_{n}, \mathfrak{\&}(n)$ and $F_{n}^{*}$ :

$$
\mathfrak{m}(n)=F_{n}+\mathfrak{z}(n)+F_{n}^{*} .
$$

We now define an isomorphism $\psi$ of $\mathfrak{m}(n)$ onto $\mathfrak{n}$ by the following formulae:

$$
\begin{aligned}
\psi(A) \xi_{0} & =-S_{R} \xi_{0}+\xi, \quad \psi(A) \xi_{\infty}=\phi^{-1} E+S_{R} \xi_{\infty}, \\
\psi(A) \eta & =\langle\eta, E) \xi_{0}+S_{0(n)} \eta+\phi(\xi, \eta) \xi_{\infty},
\end{aligned}
$$


where $A=\xi+S+E, \xi \in F_{n}, S \in \mathfrak{B}(n), E \in F_{n}^{*}$ and $\eta \in F_{n} . \psi$ being an isomorphism of $\mathfrak{m}(n)$ onto $\mathfrak{n}$, we can transfer the structure of the Lie algebra to $\mathfrak{m}(n)$ in sueh a way that $\psi$ becomes an isomorphism of Lie algebras: The bracket operation of $\mathfrak{m}(n)$ is given as follows:

(i) For $\xi, \xi^{\prime} \in F_{n}, A, B \in \mathcal{B}(n)$ and $E, E^{\prime} \in F_{n}^{*},\left[\xi, \xi^{\prime}\right]=0,\left[E, E^{\prime}\right]=0$, $[A, B]=A B-B A,[A, \xi]=A \xi,[A, E]=-{ }^{t} A E$;

(ii) For $\xi \in F_{n}$ and $E \in F_{n}^{*},[\xi, E]$ belongs to $\mathcal{B}(n):[\xi, E]_{R}=\langle\xi, E\rangle$; $[\xi, E]_{0(n)}=\langle\eta, E\rangle \xi-\phi(\xi, \eta) \phi^{-1} E$,

where $\eta$ is in $F_{n}$. In the following, we identify $\mathrm{m}(n)$, provided with this bracket operation, with the Lie algebra of $M(n)$.

(1.8) We have $\exp A=\omega(\exp \psi(A))$, that is,

$$
\exp A \cdot \omega(X)=\omega(\exp \psi(A) \cdot X) \quad \text { for all } A \in \mathfrak{m}(n) \text { and } X \in C \text {. }
$$

In particular, we have $\exp \xi \cdot o=\omega\left(\xi_{0}+\xi+\phi(\xi, \xi) / 2 \xi_{\infty}\right)$. If we set $E_{n}=M_{n}$ $-\{\infty\}$, the mapping $\xi \rightarrow \exp \xi \cdot o$ gives a one-to-one correspondence of $F_{n}$ with $E_{n}$. This mapping is known as the stereographic projection of the Euclidean space into the sphere. Finally we remark that the notation exp, introduced in (1.5), is legitimate, because we have $\exp \psi(E)=\psi((1, E))$ for each $E \in F_{n}^{*}$.

(1.9) The Lie algebra $\mathrm{m}^{\prime}(n)$ of $M^{\prime}(n)$ is given by

$$
\mathfrak{m}^{\prime}(n)=\mathfrak{o}(n)+R+F_{n}^{*} .
$$

(1.10) The decomposition of $\mathfrak{m}(n)$

$$
\mathfrak{m}(n)=F_{n}+\mathfrak{m}^{\prime}(n)
$$

is fundamental for our later considerations. For each $A \in \mathfrak{m}(n)$, we denote by $A_{F_{n}}$ and $A_{\mathfrak{m}^{\prime}(n)}$ the $F_{n^{-}}$and $\mathrm{m}^{\prime}(n)$-components of $A$ respectively. We have the following formulae on the adjoint representation of $M^{\prime}(n)$ in $\mathfrak{m}(n)$ :

$$
\begin{aligned}
& \quad \operatorname{ad} \sigma \xi=\sigma \xi ; \text { ad } \sigma E={ }^{t} \sigma^{-1} E ; \quad \text { ad }(\exp E) A=A+[E, A] ; \\
& \operatorname{ad}(\exp E) \xi=\xi+[E, \xi]+2^{-1}[E,[E, \xi]],
\end{aligned}
$$

where $\sigma \in S(n), \xi \in F_{n}, A \in \mathfrak{z}(n)$ and $E \in F_{n}^{*}$. It follows that, for each $\sigma \in M^{\prime}(n)$ and $\xi \in F_{n}, l(\sigma)=(\operatorname{ad} \sigma \xi)_{F_{n}}=$ ad $l(\sigma) \xi$, from which we see that $l$ may be considered as the homomorphism of the isotropy group $M^{\prime}(n)$ of $M(n)$ at $o$ onto the linear isotropy group.

(1.11) Let $J$ be a linear mapping of $F_{n}$ into $F_{n}^{*}$. We now define a bilinear mapping $\tilde{J}$ of $F_{n} \times F_{n}$ into $\mathfrak{g l}\left(F_{n}\right)$ (the Lie algebra of all endomorphisms of $\left.F_{n}\right)$ by

$$
\tilde{J}\left(\xi, \xi^{\prime}\right) \eta=-([\xi, J(\eta)]+[J(\xi), \eta])_{0(n)} \xi^{\prime} \quad \text { for all } \eta \in F_{n} .
$$

Later on, we shall use

Lemma 1. For any bilinear function $Q\left(\xi, \xi^{\prime}\right)$ on $F_{n} \times F_{n}$, there exists one and only one linear mapping $J$ of $F_{n}$ into $F_{n}^{*}$ such that 


$$
\operatorname{Tr}\left(\tilde{J}\left(\xi, \xi^{\prime}\right)\right)=Q\left(\xi, \xi^{\prime}\right)
$$

for all $\xi, \xi^{\prime} \in F_{n}$.

More precisely, $J$ is given by

$$
\left\langle\xi, J\left(\xi^{\prime}\right)\right\rangle=\frac{1}{n-2}\left(Q\left(\xi, \xi^{\prime}\right)-\frac{Q_{0} \cdot \phi\left(\xi, \xi^{\prime}\right)}{2(n-1)}\right),
$$

where we have set $Q_{0}=\sum_{i=1}^{n} Q\left(\xi_{i}, \xi_{i}\right),\left(\xi_{i}\right)$ being an orthogonal base of $F_{n}$ with respect to the inner product $\phi$.

2. Orthogonal bundles and conformal $S(n)$-bundles. In the following, we shall denote by $M$ a connected manifold which satisfies the second countability axiom, and we always assume that the dimension $n$ of $M$ is $\geqq 3$.

We shall say that two Riemannian metrics $g$ and $\bar{g}$ on a manifold $M$ are conformally equivalent, if there exists a positive function $\lambda$ on $M$ such that

$$
\bar{g}=\lambda^{2} g \text {. }
$$

$\lambda$ will be called the associated function of $\bar{g}$ with respect to $g$. Now, fix a Riemannian metric $g$ on $M$. A transformation $f$ of $M$ onto itself is, by definition, a conformal transformation of $g$, if $f^{*} g$ and $g$ are conformally equivalent. The group $C(g)$ of all the conformal transformations of $g$ contains the group $I(g)$ of all the isometries of $g$.

Let $M$ be a manifold and let $P_{L}$ be the bundle of frames of $M$. Then, $P_{L}$ may be considered as a principal fiber bundle over the base space $M$ with the general linear group of $F_{n}$ as structure group. Each element $x$ of $P_{L}$ gives a linear isomorphism of $F_{n}$ with $M_{p}$ such that $(x \cdot \sigma) \cdot \xi=x \cdot(\sigma \xi)$ for all $\sigma \in G L(n)$ and $\xi \in F_{n}$, where we have set $\pi_{L}(x)=p, \pi_{L}$ being the projection of $P_{L}$ onto $M$.

We know that to each Riemannian metric on $M$ there corresponds a principal fiber bundle $P_{0}$ over the base space $M$ with structure group $O(n)$ which is a subbundle of $P_{L}$. Such a bundle is usually called an orthogonal bundle. Given a Riemannian metric $g$ on $M, P_{0}$ is defined as the subset of $P_{L}$ composed of all the elements $x$ such that $g\left(x \cdot \xi, x \cdot \xi^{\prime}\right)=\phi\left(\xi, \xi^{\prime}\right)$ for all $\xi, \xi^{\prime} \in F_{n}$.

Definition 1. A conformal $S(n)$-bundle over a manifold $M$ is a principal fiber bundle over the base space $M$ with structure group $S(n)$ which is a subbundle of the bundle of frames of $M$.

We first show that to each class $\mathbb{S}$ of conformally equivalent Riemannian metrics on $M$ there corresponds a conformal $S(n)$-bundle $P_{S}$ over $M$. Let (5 be a class of conformally equivalent Riemannian metrics. Fixing an element $g$ of $\mathfrak{E}, P_{S}$ is defined as the subset of $P_{L}$ composed of all the elements $x$ as follows: There exists a real number $\rho$ such that

$$
g\left(x \cdot \xi, x \cdot \xi^{\prime}\right)=\rho \cdot \phi\left(\xi, \xi^{\prime}\right) \quad \text { for all } \xi, \xi^{\prime} \in F_{n} .
$$

Clearly $P_{S}$ does not depend on the choice of $g$ and the structure of $P_{S}$ as a principal fiber bundle is naturally induced from that of $P_{L}$. From the definition of $P_{S}$, we see that the assignment $g \rightarrow P_{0}$ gives a one-to-one correspond- 
ence of $\mathbb{S}$ with the set of all the orthogonal bundles over $M$ which are contained in $P_{S}$. In the same way, the assignment $\mathfrak{S} \rightarrow P_{S}$ gives a one-to-one. correspondence of the set of all classes of conformally equivalent Riemannian metrics with the set of all the conformal $S(n)$-bundles.

Let $P_{S}$ be a conformal $S(n)$-bundle over a manifold $M$ and let $P_{0}$ be an arbitrary orthogonal bundle contained in $P_{S}$. We define a homomorphism $s$ of $P_{S}$ onto $P_{0}$ corresponding to the homomorphism $s$ of $S(n)$ onto $O(n)$ defined in (1.4), by the requirement that $s(x)=x$ for all $x \in P_{0}$. Now consider a second Riemannian metric $\bar{g}$ which is conformally equivalent to $g$. Let $\bar{P}_{0}$ be the corresponding orthogonal bundle and let $\bar{s}$ be the corresponding homomorphism of $P_{S}$ onto $\bar{P}_{0}$. Let $\lambda$ be the associated function of $\bar{g}$ with respect to g. Then, we have

$$
s(x)=\bar{s}(x) \cdot \lambda \circ \pi_{S}(x)
$$

for all $x \in P_{S}$,

where $\pi_{S}$ denotes the projection of $P_{S}$ onto $M$ and $\lambda \circ \pi_{S}(x)$ is identified with an element of $R_{+} \subset S(n)$.

In the following, we shall give some definitions and formulations about an orthogonal bundle and connections in it which we need in the subsequent sections.

Let $P_{0}$ be an orthogonal bundle over a manifold $M$. An affine connection in $P_{0}$ (in the sense of Cartan connection) is usually called a Euclidean connection in $P_{0}$. This may be formulated as follows [5, p. 6]: A Euclidean connection in $P_{0}$ is a linear mapping $B_{0}$ of $F_{n}$ into $\mathfrak{X}\left(P_{0}\right)$ which satisfies the following conditions:

(E.1) $P_{0 x}=B_{0 x}+O(n)_{x}$, where $B_{0 x}$ denotes the subspace of $P_{0 x}$ composed of all the elements $B_{0}(\xi)_{x}$ where $\xi \in F_{n}$;

(E.2) $R_{\sigma} B_{0}(\xi)=B_{0}\left(\sigma^{-1} \xi\right)$;

(E.3) $\theta B_{0}(\xi)_{x}=\xi$, where $\theta$ denotes the $F_{n}$-valued 1 -form on $P_{0}$ defined by $\theta(X)=x^{-1} \cdot \pi_{0} X$ for all $X \in P_{0 x}$ and $x \in P_{0}$.

The assignment $x \rightarrow B_{0 x}$ defines a connection in $P_{0}$, which we call the linear connection in $P_{0}$ associated with the Euclidean connection $B_{0}$. By (E.1), there exist, for each $x \in P_{0}$, bilinear mappings $T_{x}$ and $R_{x}$ of $F_{n} \times F_{n}$ into $F_{n}$ and $\mathfrak{o}(n)$ respectively such that

$$
-\left[B_{0}(\xi), B_{0}\left(\xi^{\prime}\right)\right]_{x}=B_{0}\left(T_{x}\left(\xi, \xi^{\prime}\right)\right)_{x}+R_{x}\left(\xi, \xi^{\prime}\right)_{x}^{*} .
$$

$T_{x}$ (resp. $R_{x}$ ) corresponds to the torsion (resp. the curvature) tensor field of the Euclidean connection. There exists one and only one Euclidean connection in $P_{0}$, called the Riemannian connection in $P_{0}$, such that $T_{x}=0$ for all $x \in P_{0}$.

Let $B_{0}$ be a Euclidean connection in $P_{0}$. We now define, for each $x \in P_{0}$, a bilinear function $S_{x}$ on $F_{n} \times F_{n}$ by

$$
S_{x}\left(\xi, \xi^{\prime}\right)=\operatorname{Tr}\left(\tilde{R}_{x}\left(\xi, \xi^{\prime}\right)\right),
$$


where $\widetilde{R}_{x}$ denotes the bilinear mapping of $F_{n} \times F_{n}$ into $\mathfrak{g l}\left(F_{n}\right)$ defined by $\tilde{R}_{x}\left(\xi, \xi^{\prime}\right) \eta=R_{x}(\xi, \eta) \xi^{\prime}$ for all $\eta, \xi, \xi^{\prime} \in F_{n}$. If we denote by $S(X, Y)$ the ordinary Ricci tensor field of the Euclidean connection, then we have

$$
S_{x}\left(\xi, \xi^{\prime}\right)=S\left(x \cdot \xi, x \cdot \xi^{\prime}\right) .
$$

Finally, we define, for each $x \in P_{0}$, a linear mapping $J_{x}$ of $F_{n}$ into $F_{n}^{*}$ by the formula

$$
\left\langle\xi, J_{x}\left(\xi^{\prime}\right)\right\rangle=\frac{1}{n-2}\left(S_{x}\left(\xi, \xi^{\prime}\right)-\frac{S_{0}(x)}{2(n-1)} \phi\left(\xi, \xi^{\prime}\right)\right),
$$

where $S_{0}(x)=\sum_{i=1}^{n} S_{x}\left(\xi_{i}, \xi_{i}\right),\left(\xi_{i}\right)$ being an orthogonal base of $F_{n} . S_{0}$ corresponds to what is usually called the scalar curvature.

3. Conformal $M^{\prime}(n)$-bundle associated with a conformal $S(n)$-bundle.

Proposition 1. For each conformal $S(n)$-bundle $P_{S}$ over a manifold $M$ there exists a collection $\left(P^{\prime}, l, \alpha\right)$ as follows: $P^{\prime}$ is a principal fiber bundle over the base space $M$ with structure group $M^{\prime}(n) ; l$ is a homomorphism of $P^{\prime}$ onto $P_{S}$ corresponding to the homomorphism l of $M^{\prime}(n)$ onto $S(n)$ defined in (1.5); $\alpha$ is an $R$-valued 1-form on $P^{\prime}$ having the following two properties:

(i) $\alpha\left(A^{*}\right)=A_{R}$ for all $A \in \mathfrak{m}^{\prime}(n)$, where $A_{R}$ denotes the $R$-component of $A$ in the decomposition of $\mathrm{m}^{\prime}(n)$ given in (1.9);

(ii) $R_{\sigma}^{*} \alpha=\alpha+\left[\operatorname{ad} l(\sigma)^{-1} \theta, E(\sigma)\right]_{R}$ for all $\sigma \in M^{\prime}(n)$, where $\theta$ denotes the $F_{n}$-valued 1-form on $P^{\prime}$ defined by $\theta(X)=l(z)^{-1} \pi X$ for each $X \in P_{z}^{\prime}$ and $z \in P^{\prime}$, $\pi$ being the projection of $P^{\prime}$ onto $M$, and where $\sigma=l(\sigma) \cdot \exp E(\sigma), E(\sigma) \in F_{n}^{*}$.

Proof. Fix an orthogonal bundle $P_{0} \subset P_{S}$ and let $s$ be the homomorphism of $P_{S}$ onto $P_{0}$ defined in $\S 2$. By making use of $P_{0}$ and the injection of $O(n)$ into $M^{\prime}(n)$, we get a principal fiber bundle $P^{\prime}$ over the base space $M$ with structure group $M^{\prime}(n)$ together with an injection $h$ of $P_{0}$ into $P^{\prime}$. Next, we define $l$ to be the homomorphism of $P^{\prime}$ onto $P_{S}$ such that $l \circ h(x)=x$ for all $x \in P_{0} . \alpha$ is defined as follows: Since, for each $z \in P^{\prime}, z$ and $h \circ s \circ l(z)$ lie in the same fiber of $P^{\prime}$, it follows that there is a mapping $a$ of $P^{\prime}$ into $M^{\prime}(n)$ such that $z=h \circ s \circ l(z) \cdot a(z)$. By applying $s \circ l$ to this formula and using the fact that $l \circ h(x)=x$ for all $x \in P_{0}$, we have $s(a(z))=e$, from which it follows that there exist mappings $\rho$ and $E$ of $P^{\prime}$ into $R_{+}$and $F_{n}^{*}$ respectively such that $a(z)=\rho(z) \cdot \exp E(z)$. Now define $\alpha$ to be $\alpha=\rho^{-1} d \rho+[\theta, E]_{R}$. That the form $\alpha$, just defined, has the properties (i) and (ii) follows from the following Lemma 2 .

\section{LEMMA 2.}

(i) $\rho(z \cdot \sigma)=\rho(z) \cdot \rho \circ l(\sigma), E(z \cdot \sigma)=\operatorname{ad} l(\sigma)^{-1} E(z)+E(\sigma)$;

(ii) $R_{\sigma}^{*} \theta=\operatorname{ad} l(\sigma)^{-1} \theta, \theta\left(A^{*}\right)=0$.

Lemma 3. Let $V$ be a manifold and let $f$ and $g$ be two mappings of $V$ into $P^{\prime}$. A ssume that there exist mappings $\rho$ and $E$ of $V$ into $R_{+} \subset M^{\prime}(n)$ and $F_{n}^{*} \subset \mathfrak{m}^{\prime}(n)$ respectively such that 


$$
f(u)=g(u) \cdot \rho(u) \cdot \exp E(u)
$$

Then, for each $u \in V$ and $X \in V_{u}$, we get

$$
f X=R_{a(u)} \circ g X+\left(\rho^{-1} d \rho(X)\right)_{f(u)}^{*}+\left(\left[\rho^{-1} d \rho(X), E(u)\right]+b^{-1} d b(X)\right)_{f(u)}^{*},
$$

where $a(u)=\rho(u) \cdot \exp E(u)$ and $b(u)=\exp E(u) \cdot \rho^{-1} d \rho\left(\right.$ resp. $\left.b^{-1} d b\right)$ is an $R$-(resp. $\left.F_{n^{-}}^{*}\right)$ valued 1 -form on $V$.

Proposition 2. Let $\left(P^{\prime}, l, \alpha\right)$ be a collection having the properties in Proposition 1. Then, for each orthogonal bundle $P_{0} \subset P_{S}$ there exists one and only one injection $h$ of $P_{0}$ into $P^{\prime}$ such that

(i) $l \circ h(x)=x$ for all $x \in P_{0}$;

(ii) $h^{*} \alpha=0$.

Proof. We first show that there exists at least one injection, say $h_{0}$, of $P_{0}$ into $P^{\prime}$ such that $l \circ h_{0}(x)=x$ for all $x \in P_{0}$. For this purpose, we introduce a fiber bundle, denoted by $P^{\prime} / S(n)$, over $M$, which is the quotient space by the following equivalence relation $\sim$ in $P^{\prime}: z^{\prime} \sim z$ if and only if there is a $\tau \in S(n)$ such that $z^{\prime}=z \cdot \tau$. The standard fiber of $P^{\prime} / S(n)$ is given by $M^{\prime}(n) / S(n)$ (the space of left cosets of $M^{\prime}(n)$ modulo $\left.S(n)\right)$, which is obviously homeomorphic to a Euclidean space; hence $P^{\prime} / S(n)$ admits a (differentiable) section $g$ over $M$. Now denoting by $p$ the projection of $P^{\prime}$ onto $P^{\prime} / S(n)$, we see that, for each $x \in P_{0}$, there is a unique element $h_{0}(x)$ of $P^{\prime}$ such that $l\left(h_{0}(x)\right)=x$ and $p\left(h_{0}(x)\right)=g(\pi x)$; clearly the correspondence $x \rightarrow h_{0}(x)$ defines an injection of $P_{0}$ into $P^{\prime}$ such that $l \circ h_{0}(x)=x$ for all $x \in P_{0}$, which proves our assertion.

Now we shall prove the existence of $h$ having the properties (i) and (ii). Let $B_{0}$ be the Riemannian connection in $P_{0}$. Defining a mapping $E$ of $P_{0}$ into $F_{n}^{*}$ by $\langle\xi, E(x)\rangle=[\xi, E(x)]_{R}=-h^{*} \alpha\left(B_{0}(\xi)_{x}\right)$ for all $x \in P_{0}$ and $\xi \in F_{n}$, we define $h$ by $h(x)=h_{0}(x) \cdot \exp E(x)$. First of all, we see that $l \circ h(x)=x$ for all $x \in P_{0}$. By applying Lemma 3 to the case where $V=P_{0}, f=h, g=h_{0}$ and $X=B_{0}(\xi)_{x}$, we have

$$
h B_{0}(\xi)_{x}=R_{b(x)} \circ h_{0} B_{0}(\xi)_{x}+\left(b^{-1} d b\left(B_{0}(\xi)_{x}\right)\right)_{h(x)}^{*},
$$

where $b(x)=\exp E(x)$. Applying $\alpha$ to this formula and using the properties of $\alpha$ and the fact that $h_{0}^{*} \theta\left(B_{0}(\xi)_{x}\right)=\xi$, we have

$$
h^{*} \alpha\left(B_{0}(\xi)_{x}\right)=h_{0}^{*} \alpha\left(B_{0}(\xi)_{x}\right)+[\xi, E(x)]_{R}=0 ;
$$

furthermore, we have $h^{*} \alpha\left(A_{x}^{*}\right)=A_{R}=0$ for all $A \in \mathfrak{o}(n)$. It follows immediately that $h^{*} \alpha=0$.

Now assume that there exist two injections $h$ and $h_{0}$ which both have the properties (i) and (ii). It follows from property (i) that there is a mapping $E$ of $P_{0}$ into $F_{n}^{*}$ such that $h(x)=h_{0}(x) \cdot \exp E(x)$ for all $x \in P_{0}$. Then, by the first equality of (3.1), we see that $\langle\xi, E(x)\rangle=0$ for all $\xi \in F_{n}$ and $x \in P_{0}$, that is, $E(x)=0$, whence $h=h_{0}$. q.e.d. 
Proposition 3. Let $\left(P^{\prime}, l, \alpha\right)$ be a collection having the properties in Proposition 1. Let $P_{0}$ be an orthogonal bundle contained in $P_{S}$ and let $h$ be the corresponding injection of $P_{0}$ into $P^{\prime}$ whose existence is assured by Proposition 2. Then, there exist mappings $\rho$ and $E$ of $P^{\prime}$ into $R_{+}$and $F_{n}^{*}$ respectively such that

$$
z=h \circ s \circ l(z) \cdot \rho(z) \cdot \exp E(z)
$$

and, using these $\rho$ and $E, \alpha$ is expressed as

$$
\alpha=\rho^{-1} d \rho+[\theta, E]_{R} \text {. }
$$

Proof. By applying Lemma 3 to the case where $V=P^{\prime}, f=$ the identity transformation of $P^{\prime}$ onto itself, $g=h \circ s \circ l$ and $u=z$, we obtain

$$
X=R_{a(z)} \circ h \circ s \circ l X+\left(\rho^{-1} d \rho(X)\right)_{z}^{*}+\left(\left[\rho^{-1} d \rho(X), E(z)\right]+b^{-1} d b(X)\right)_{z}^{*} .
$$

Applying $\alpha$ to this formula and using the properties of $\alpha$ and the fact that $h^{*} \alpha=0$, we have $\alpha(X)=\left[\operatorname{ad} \rho(z)^{-1} \theta(h \circ s \circ l X), E(z)\right]_{R}+\rho^{-1} d \rho(X)$. By an analogous argument, we have $\theta(X)=\operatorname{ad} \rho(z)^{-1} \theta(h \circ s \circ l X)$. q.e.d.

By Propositions 2 and 3 we have easily

Proposition 4. Let $\left(P^{\prime}, l, \alpha\right)$ and $\left(\bar{P}^{\prime}, \bar{l}, \bar{\alpha}\right)$ be two collections both having the properties in Proposition 1 for the same conformal $S(n)$-bundle $P_{S}$. Then, there exists a unique isomorphism $f$ of $\bar{P}^{\prime}$ onto $P^{\prime}$ such that $l \circ f=\bar{l}$ and $f^{*} \alpha=\bar{\alpha}$.

These observations lead us to the following

Definition 2. Let $P_{S}$ be a conformal $S(n)$-bundle over a manifold $M$. The conformal $M^{\prime}(n)$-bundle associated with $P_{S}$ is the principal fiber bundle $P^{\prime}$ together with a homomorphism $l$ and a form $\alpha$ having the properties in Proposition $1[1 ; 7 ; 3 ; 2]$.

The prototype of conformal $M^{\prime}(n)$-bundles is given by the Möbius space $M_{n}$ : By the homogeneous structure of $M_{n}=M(n) / M^{\prime}(n)$, the Möbius group $M(n)$ may be considered as a principal fiber bundle $P^{\prime}$ over the base space $M_{n}$ with structure group $M^{\prime}(n)$. At the same time, since the linear isotropy group of $M(n)$ at $o$ is identified with $S(n)$, the homogeneous structure yields a conformal $S(n)$-bundle $P_{S}$ over $M_{n}$ together with a homomorphism $l$ of $P^{\prime}$ onto $P_{S}$. Moreover, we have a left invariant form $\alpha$ on $P^{\prime}(=M(n))$ such that $\alpha(X)=X_{R}$ for all $X \in \mathrm{m}(n)$, where $X_{R}$ denotes the $R$-component of $X$ in the decomposition of $\mathfrak{m}(n)$ given in (1.6) and (1.7), and it can be proved that it has the properties stated in Proposition 1. Therefore, we see that $P^{\prime}$ together with $l$ and $\alpha$, obtained in this way, gives the conformal $M^{\prime}(n)$-bundle associated with $P_{S}$, and it is considered as the prototype of conformal $M^{\prime}(n)$ bundles.

REMARK. Let $P_{S}$ be a conformal $S(n)$-bundle over a manifold $M$ and let $P^{\prime}$ together with $l$ and $\alpha$ be the corresponding conformal $M^{\prime}(n)$-bundle. Given an orthogonal bundle $P_{0} \subset P_{S}$, the existence of the injection $h$ of $P_{0}$ into $P^{\prime}$ assured by Proposition 2 corresponds to that of the so-called "Veblen's 
repère" which is uniquely determined by the Riemannian metric on $M$ which corresponds to $P_{0}$ and by a choice of a coordinate system $[7 ; 3]$.

Let $P^{\prime}$ be a conformal $M^{\prime}(n)$-bundle over a manifold $M$. For each point $p$ of $M$, the fiber over $p$ of the associated fiber bundle of $P^{\prime}$ with standard fiber $M_{n}$ is called the tangent Möbius space at $p$, which we shall denote by $M_{n}(p)$. Every element $z$ of $P^{\prime}$ gives a one-to-one correspondence of $M_{n}$ with $M_{n}(p)$ such that $(z \cdot \sigma) \cdot u=z \cdot(\sigma u)$ for all $\sigma \in M^{\prime}(n)$ and $u \in M_{n}$, where $\pi(z)=p$. The origin $p^{*}$ of the tangent Möbius space at $p$ is the point of $M_{n}(p)$ defined by $p^{*}=z \cdot o$, where $z$ is an element of $P^{\prime}$ such that $\pi(z)=p$. Clearly, the definition is consistent. By making use of $P^{\prime}$ and the injection of $M^{\prime}(n)$ into $M(n)$, we define a principal fiber bundle $P$ over the base space $M$ with structure group $M(n)$. In this case, we identify $P^{\prime}$ with a subbundle of $P$.

Finally we have the following diagram:

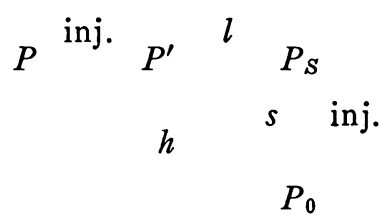

4. Normal conformal connection associated with a conformal $S(n)$-bundle.

Proposition 5. Let $P_{S}$ be a conformal $S(n)$-bundle over a manifold $M$ and let $P^{\prime}$ together with $l$ and $\alpha$ be the corresponding conformal $M^{\prime}(n)$-bundle. Then, there exists a unique linear mapping $B$ of $F_{n}$ into $\mathfrak{X}\left(P^{\prime}\right)$ which satisfies the following conditions:

(C.1) $B_{z}+M^{\prime}(n)_{z}=P_{z}^{\prime}$ (direct sum), where $B_{z}$ denotes the subspace of $P_{z}^{\prime}$ composed of all the elements $B(\xi)_{z}$ where $\xi$ runs over $F_{n}$;

(C.2) $R_{\sigma} B(\xi)=B\left(\left(\operatorname{ad} \sigma^{-1} \xi\right)_{F_{n}}\right)+\left(\operatorname{ad} \sigma^{-1} \xi\right)_{\mathfrak{m}^{\prime}(n)}^{*}$;

(C.3) $\theta B(\xi)=\xi$, where $\theta$ denotes the $F_{n}$-valued 1 -form on $P^{\prime}$ defined in Proposition 1 ;

(C.4) $\alpha B(\xi)=0$;

(C.5) For each orthogonal bundle $P_{0} \subset P_{S}$, let $h$ be the corresponding injection of $P_{0}$ into $P^{\prime}$. Then we have

$$
h B_{0}(\xi)_{x}=B(\xi)_{h(x)}+J_{x}(\xi)_{h(x)}^{*},
$$

where $B_{0}$ denotes the Riemannian connection in $P_{0}$ associated with $P_{0}$ and $J_{x}$ the linear mapping of $F_{n}$ into $F_{n}^{*}$ given by formula (2.2).

The above proposition corresponds to Proposition 1 of [5] which is concerned with the normal projective connection associated with a class of projectively equivalent affine connections. One will see that the proof of Proposition 5 is parallel to that of Proposition 1 cited above.

Proof of Proposition 5. The proof is divided into three steps.

I. A linear mapping $B$ of $F_{n}$ into $\mathfrak{X}\left(P^{\prime}\right)$ will be called a conformal connec- 
tion in the conformal $M^{\prime}(n)$-bundle $P^{\prime}$ if it satisfies conditions (C.1), (C.2), (C.3) and (C.4) (see Definition 3).

Let $B$ be a conformal connection in $P^{\prime}$. We see from condition (C.1) that, for each $z \in P^{\prime}$, there exist bilinear mappings $T_{z}$ and $A_{z}$ of $F_{n} \times F_{n}$ into $F_{n}$ and $\mathfrak{m}^{\prime}(n)$ respectively such that

$$
-\left[B(\xi), B\left(\xi^{\prime}\right)\right]_{z}=B\left(T_{z}\left(\xi, \xi^{\prime}\right)\right)_{z}+A_{z}\left(\xi, \xi^{\prime}\right)_{z}^{*} .
$$

Denoting by $W_{z}\left(\xi, \xi^{\prime}\right)$ the $\mathfrak{o}(n)$-component of $A_{z}\left(\xi, \xi^{\prime}\right)$ in the decomposition of $\mathfrak{m}^{\prime}(n)$ given in (1.9), we have the following two formulae (cf. $[5$, p. 8]).

(i) $T_{z \cdot \sigma}\left(\xi, \xi^{\prime}\right)=\operatorname{ad} l(\sigma)^{-1} T_{z}\left(\operatorname{ad} l(\sigma) \xi, \operatorname{ad} l(\sigma) \xi^{\prime}\right)$;

(ii) If $T_{z}=0 \quad$ for all $z \in F^{\prime}$,

$$
W_{z \cdot \sigma}\left(\xi, \xi^{\prime}\right)=\operatorname{ad} l(\sigma)^{-1} W_{z}\left(\operatorname{ad} l(\sigma) \xi, \operatorname{ad} l(\sigma) \xi^{\prime}\right) .
$$

Let $P_{0}$ be an orthogonal bundle contained in $P_{S}$ and let $h$ be the corresponding injection of $P_{0}$ into $P^{\prime}$. We know from Proposition 3 that there exist mappings $\rho$ and $E$ of $P^{\prime}$ into $R_{+}$and $F_{n}^{*}$ respectively such that

$$
z=h \circ \operatorname{sol}(z) \cdot \rho(z) \cdot \exp E(z) \text {. }
$$

By applying Lemma 3 to the case where $V=P^{\prime}, f=$ the identity transformation of $P^{\prime}$ onto itself, $g=h \circ s \circ l$ and $X=B(\xi)_{h(x)}$, we have

$$
B(\xi)_{h(x)}=h \circ s \circ l B(\xi)_{h(x)}+A_{x}(\xi)_{h(x)}^{*}-J_{x}(\xi)_{h(x)}^{*},
$$

where we have set $A_{x}(\xi)=\rho^{-1} d \rho\left(B(\xi)_{h(x)}\right)$ and $J_{x}(\xi)=-b^{-1} d b\left(B(\xi)_{h(x)}\right)$. By condition (C.4) and the formula of $\alpha$ in Proposition 3, we have $A_{x}(\xi)=0$ for all $\xi \in F_{n}$. If we define a linear mapping $B_{0}$ of $F_{n}$ into $\mathfrak{X}\left(P_{0}\right)$ by $B_{0}(\xi)_{x}$ $=s \circ l B(\xi)_{h(x)}$, then we see from conditions (C.1), (C.2) and (C.3) that it satisfies conditions (E.1), (E.2) and (E.3) for a Euclidean connection in $P_{0}$. The Euclidean connection $B_{0}$ in $P_{0}$ which is obtained in this way will be said to be induced by $P_{0}$ (with respect to the conformal connection $B$ ). For each $x \in P_{0}$, the correspondence $\xi \rightarrow J_{x}(\xi)$ defines a linear mapping of $F_{n}$ into $F_{n}^{*}$, which will be said to be induced by $P_{0}$. With the above definition, we have

$$
h B_{0}(\xi)_{x}=B(\xi)_{h(x)}+J_{x}(\xi)_{h(x)}^{*} .
$$

Let $P_{0}$ be an orthogonal bundle contained in $P_{S}$ and let $B_{0}$ (resp. $J_{x}$ ) be the Euclidean connection in $P_{0}$ (resp. the linear mapping of $F_{n}$ into $F_{n}^{*}$ ) induced by $P_{0}$. As we have observed in $\S 2$, we can take, for each $x \in P_{0}$, bilinear mappings $T_{x}^{\prime}$ and $R_{x}$ of $F_{n} \times F_{n}$ into $F_{n}$ and $\mathrm{o}(n)$ respectively such that

$$
-\left[B_{0}(\xi), B_{0}\left(\xi^{\prime}\right)\right]_{x}=B_{0}\left(T_{x}^{\prime}\left(\xi, \xi^{\prime}\right)\right)_{x}+R_{x}\left(\xi, \xi^{\prime}\right)_{x}^{*} .
$$

$T_{x}^{\prime}$ (resp. $R_{x}$ ) corresponds to the torsion (resp. the curvature) tensor field of $B_{0}$. Then, we have 

(i) $T_{h(x)}\left(\xi, \xi^{\prime}\right)=T_{x}^{\prime}\left(\xi, \xi^{\prime}\right)$;
(ii) $W_{h(x)}\left(\xi, \xi^{\prime}\right)=R_{x}\left(\xi, \xi^{\prime}\right)+\left(\left[\xi, J\left(\xi^{\prime}\right)\right]+\left[J_{x}(\xi), \xi^{\prime}\right]\right)_{0(n)}$

(cf. $[5$, p. 10]).

II. Let $B$ be a conformal connection in $P^{\prime}$. Now consider the following condition for $B$ :

(i) $T_{z}\left(\xi, \xi^{\prime}\right)=0$;

(ii) $\operatorname{Tr}\left(\tilde{W}_{z}\left(\xi, \xi^{\prime}\right)\right)=0$,

where $\widetilde{W}_{z}\left(\xi, \xi^{\prime}\right)$ denotes the bilinear mapping of $F_{n} \times F_{n}$ into $\mathfrak{g l}\left(F_{n}\right)$ defined by $\tilde{W}_{z}\left(\xi, \xi^{\prime}\right) \eta=W_{z}(\xi, \eta) \xi^{\prime}$ for all $\eta \in F_{n}$.

We shall show that, if a conformal connection in $P^{\prime}$ satisfies condition $\left(\mathrm{C} .5^{\prime}\right)$, then it satisfies also condition (C.5). Let $P_{0}$ be an orthogonal bundle contained in $P_{S}$ and let $B_{0}$ (resp. $J_{x}$ ) be the Euclidean connection in $P_{0}$ (resp. the linear mapping of $F_{n}$ into $F_{n}^{*}$ ) induced by $P_{0}$. By formula (4.2), it is sufficient to show that $B_{0}$ is the Riemannian connection in $P_{0}$ and $J_{x}$ is given by formula (2.2). It follows from formula (i) of (4.3) that $T_{x}^{\prime}\left(\xi, \xi^{\prime}\right)=T_{h(x)}\left(\xi, \xi^{\prime}\right)$ $=0$, which shows that $B_{0}$ is the Riemannian connection in $P_{0}$. Formula (ii) of (4.3) can be written as

$$
\tilde{W}_{h(x)}\left(\xi, \xi^{\prime}\right)=\widetilde{R}_{x}\left(\xi, \xi^{\prime}\right)-\tilde{J}_{x}\left(\xi, \xi^{\prime}\right) ;
$$

if we observe that $\operatorname{Tr}\left(\widetilde{R}_{x}\left(\xi ; \xi^{\prime}\right)\right)=S_{x}\left(\xi, \xi^{\prime}\right)$, formula (ii) of (C.5') gives $\operatorname{Tr}\left(\tilde{J}_{x}\left(\xi, \xi^{\prime}\right)\right)=S_{x}\left(\xi, \xi^{\prime}\right)$. Now, by Lemma 1 , we see that $J_{x}$ is given by formula (2.2), which proves our assertion.

III. Now we can prove Proposition 5.

We first prove the uniqueness of $B$ : Fix an orthogonal bundle $P_{0} \subset P_{S}$ and let $h$ be the corresponding injection of $P_{0}$ into $P^{\prime}$. By Proposition 3, we can take mappings $\rho$ and $E$ such that $z=h \circ s \circ l(z) \cdot \rho(z) \cdot \exp E(z)$. Now, by condition (C.2), we have easily

$$
B(\xi)_{z}=R_{a(z)} B(\operatorname{ad} \rho(z) \xi)_{h(x)}-\left(\left(\operatorname{ad} b(z)^{-1} \xi\right)_{\mathfrak{m}(n)}\right)_{z}^{*}
$$

where $x=s \circ l(z)$ and $b(z)=\exp E(z)$. If we take account of the formula $B(\xi)_{h(x)}=h B_{0}(\xi)_{x}-J_{x}(\xi)_{h,(x)}^{*}$, formula (4.5) implies that $B$ is uniquely determined by the orthogonal bundle $P_{0}$ only, which proves the uniqueness of $B$.

We shall now prove the existence of $B$ : Fix an orthogonal bundle $P_{0} \subset P_{S}$ and let $h$ be the corresponding injection of $P_{0}$ into $P^{\prime}$. Let $B_{0}$ be the Riemannian connection in $P_{0}$ and define $J_{x}$ by formula (2.2). We now define a linear mapping $B$ of $F_{n}$ into $\mathfrak{X}\left(P^{\prime}\right)$ in the following way. First, we define, for each $x \in P_{0}$ and $\xi \in F_{n}, B(\xi)_{h(x)}$ by the formula $B(\xi)_{h(x)}=h B_{0}(\xi)_{x}-J_{x}(\xi)_{h(x)}^{*}$. Next, we define, for each $z \in P^{\prime}$ and $\xi \in F_{n}, B(\xi)_{z}$ by formula (4.5) by the use of $B(\xi)_{h(x)}$, just defined. Then, the linear mapping $B$ which is obtained in this way satisfies the required conditions. In fact, by condition (E.2) and the fact that $J_{x \cdot \tau}(\xi)={ }^{t} \tau J_{x}(\tau \xi)$ for all $x \in P_{0}$ and $\tau \in O(n)$, we have easily $R_{\tau} B(\xi)_{h(x)}$ 
$=B\left(\operatorname{ad} \tau^{-1} \xi\right)_{h(x \cdot \tau)}$. Therefore, we see that condition (C.2) follows from the following two formulae $\rho(z \cdot \sigma)=\rho(z) \cdot \rho \circ l(\sigma)$ and $b(z) \cdot \sigma=l(\sigma) \cdot b(z \cdot \sigma)$ (see Lemma 2 ). In order to verify condition (C.1), it is sufficient to deal with the case where $z$ is of the form $z=h(x)$. From the proof of Proposition 3, we know that, for each $X \in P_{h(x)}^{\prime}$, there exist a $Y \in P_{0 x}$ and an $A \in \mathfrak{m}^{\prime}(n)$ such that $X=h Y+A_{h(x)}^{*}$. We now see that condition (C.1) follows from condition (E.1). Condition (C.3) is obvious from condition (E.3). Condition (C.4) follows from the properties of $\alpha$. Therefore, we have only to check condition (C.5). For this purpose, it is sufficient, by the argument in II, to show that $B$ satisfies condition (C.5'). By definition of $B$ we see that $B_{0}$ and $J_{x}$ coincide with the ones induced by $P_{0}$ with respect to the conformal connection $B$. From formula (i) of (4.3), we have $T_{h(x)}\left(\xi, \xi^{\prime}\right)=0$, because $T_{x}^{\prime}\left(\xi, \xi^{\prime}\right)=0$ (note that $B_{0}$ is the Riemannian connection in $P_{0}$ ). It follows from formula (i) of (4.1) that $T_{z}\left(\xi, \xi^{\prime}\right)=0$. Moreover, by Lemma 1 , we have $\operatorname{Tr}\left(\tilde{J}_{x}\left(\xi, \xi^{\prime}\right)\right)=S_{x}\left(\xi, \xi^{\prime}\right)$ and, using formula (4.4), we have $\operatorname{Tr}\left(\tilde{W}_{h(x)}\left(\xi, \xi^{\prime}\right)\right)=0$. It follows from formula (ii) of (4.1) that $\operatorname{Tr}\left(\tilde{W}_{z}\left(\xi, \xi^{\prime}\right)\right)=0$; thus $B$ satisfies condition (C.5'). Therefore we have completed the proof of Proposition 5.

REMARK. The bilinear mapping $T_{z}\left(\right.$ resp. $W_{z}$ ) which appears in the proof of Proposition 5 corresponds to what is usually called the torsion tensor field (resp. the Weyl's conformal curvature tensor field) of the conformal connection $B$.

Remark. The proof of Proposition 5 shows that, given a conformal connection $B$ in $P^{\prime}$, conditions (C.5) and (C.5') are mutually equivalent.

Definition 3. Let $P_{S}$ be a conformal $S(n)$-bundle over a manifold $M$ and let $P^{\prime}$ together with $l$ and $\alpha$ be the corresponding conformal $M^{\prime}(n)$-bundle. A conformal connection in $P^{\prime}$ is a linear mapping $B$ of $F_{n}$ into $\mathfrak{X}\left(P^{\prime}\right)$ which satisfies conditions (C.1), (C.2), (C.3) and (C.4) in Proposition 5. The normal conformal connection associated with the conformal $S(n)$-bundle $P_{S}$ is the conformal connection $B$ which satisfies condition (C.5) in Proposition 5 or, equivalently, condition $\left(\mathrm{C} .5^{\prime}\right)$ in the proof of Proposition $5[1 ; 7 ; 3 ; 2]$.

5. Conformal development. This section corresponds to $\$ 5$ of [5], in which we proved a proposition about projective development. For the definition and fundamental properties of a connection in a principal fiber bundle, we follow the book of K. Nomizu [6].

Let $P_{S}$ be a conformal $S(n)$-bundle over a manifold $M$. Let $P^{\prime}$ be the corresponding conformal $M^{\prime}(n)$-bundle and let $P$ be the principal fiber bundle over the base space $M$ with structure group $M(n)$ defined in $\S 3$. As is well known, a conformal connection $B$ in $P^{\prime}$ gives rise to a connection $Q$ in $P$. The horizontal space $Q_{z}$ at a point $z$ of $P^{\prime}$ is given by the subspace of $P_{z}$ composed of all the elements $B(\xi)_{z}-\xi_{z}^{*}$ where $\xi$ runs over $F_{n} \subset \mathfrak{m}(n)$ (note that $P^{\prime}$ is identified with a subspace of $P$ ).

We now define conformal development as follows: Let $p$ be a point of $M$ and let $u(t)$ be a curve in $M$ beginning at $p$. Let $z(t)$ be a horizontal curve in 
$P$ which covers $u(t)$, with respect to the connection $Q$ in $P$. There is a curve $a(t)$ in $M(n)$ such that $z(t) \cdot a(t) \in P^{\prime}$ for all $t$. Then the conformal development of $u(t)$ at $p$ is defined as the curve $u^{*}(t)=z(0) \cdot a(t) o$ in the tangent Möbius space $M_{n}(p)$ at $p$. Clearly the definition is consistent.

We recall here the definition of Euclidean development, cf. [5, p. 14]. Let $B_{0}$ be a Euclidean connection in an orthogonal bundle $P_{0}$ over $M$. Let $p$ be a point of $M$ and let $u(t)$ be any curve in $M$ beginning at $p$. Let $x(t)$ be a horizontal curve in $P_{0}$ which covers $u(t)$, with respect to the linear connection associated with the Euclidean connection $B_{0}$. There is a curve $\xi(t)$ in $F_{n}$ such that $B_{0}(\xi(t))_{x(t)}=x^{\prime}(t)$. Now the Euclid development of $u(t)$ at $p$ is defined as the curve $v(t)=x(0) \cdot w(t)$ in the tangent Euclidean space $M_{p}$ at $p$, where $\boldsymbol{w}(t)=\int_{0}{ }^{t} \xi(t) d t$.

The following Proposition 6 may be considered as an alternative description of conformal development in terms of a Euclidean connection.

Let $P_{S}$ be a conformal $S(n)$-bundle over a manifold $M$ and let $P^{\prime}$ be the corresponding conformal $M^{\prime}(n)$-bundle. Let $P_{0}$ be any orthogonal bundle and let $h$ be the corresponding injection of $P_{0}$ into $P^{\prime}$. For each $x \in P_{0}$, we define $J_{x}$ by formula (2.2) starting with the Riemannian connection in $P_{0}$. Moreover, we identify $M_{n}$ (resp. $F_{n}$ ) with the tangent Möbius space $M_{n}(p)$ (resp. the tangent Euclidean space $M_{p}$ ) by $h(x)$ (resp. $x$ ). With these preparations, we have

Proposition 6. Let $p$ be a point of $M$ and let $u(t)$ be a curve in $M$ beginning at p. Fix a point $x$ of $P_{0}$ such that $\pi_{0}(x)=p$. Let $u^{*}(t)($ resp. $v(t))$ be the development of $u(t)$ at $p$ into the tangent Möbius space $M_{n}$ (resp. the tangent Euclidean space $F_{n}$ ) at $p$ with respect to the normal conformal connection in $P^{\prime}$ (resp. the Riemannian connection in $\left.P_{0}\right)$. Then, we have $u^{*}(t)=a(t) o$, where $a(t)$ denotes the curve in the Möbius group $M(n)$ which is determined by the differential equation

$$
a(t)^{-1} a^{\prime}(t)=v^{\prime}(t)+J_{x(t)}\left(v^{\prime}(t)\right)
$$

with the initial condition $a(0)=e$, where $x(t)$ denotes the lift of $u(t)$ through $x$ with respect to the linear connection associated with the Riemannian connection in $P_{0}$.

The proof of this proposition is quite similar to that of Proposition 2 of [5]. We here remark the following point only. Let $z(t)$ be the lift of $u(t)$ through $h(x)$ with respect to the connection in $P$ which corresponds to the normal conformal connection in $P^{\prime}$. Then, we have

$$
z(t) \cdot a(t)=h(x(t)) .
$$

6. Some properties of a complete Riemannian space of type $S$. Let $g$ be a Riemannian metric on a manifold $M$. By a field of projections of rank $m$ we shall mean a tensor field $H$ of type $(1,1)$ which satisfies the following 
conditions: (i) $H$ is parallel with respect to the Riemannian connection, (ii) $H^{2}=H$, (iii) $g(H X, Y)=g(X, H Y)$ for all tangent vectors $X, Y$ and (iv) the rank of $H_{p}$ is equal to $m$ at each point $p$ of $M$.

Definition 4. Let $M$ be an n-dimensional manifold.

(i) A Riemannian metric $g$ on $M$ is of type $\subseteq^{m-1}(0 \leqq m \leqq n)$, if there exists a field $H^{\prime}$ of projections of rank $m$ and if the Ricci tensor field $S$ of $g$ is expressed as

$$
S=(m-1) g \circ H^{\prime}-(n-m-1) g \circ H^{\prime \prime},
$$

where $H^{\prime \prime}$ denotes the tensor field of type $(1,1)$ defined by $H^{\prime \prime} X=X-H^{\prime} X$ for all tangent vectors $X$.

(ii) A Riemannian metric $g$ on $M$ is of type $\mathfrak{S}^{\infty}$, if the Ricci tensor field is zero. When a Riemannian metric $g$ on $M$ is of type $\widetilde{S}^{m-1}$ or $\mathbb{S}^{\infty}$, we shall say that it is of type $\mathfrak{S}$.

From the definition, we see that a Riemannian space of type $\mathbb{S}^{-1}$ (resp. $\mathfrak{S}^{n-1}$ ) is nothing but an Einstein space with the negative (resp. positive) Ricci tensor field, and that, in general, a Riemannian space of type $\widetilde{S}^{m-1}$ is locally isomorphic to the direct product of two Einstein spaces with the Ricci tensor fields of different signs. Riemannian spaces of type $\subseteq$ have been studied by S. Sasaki and K. Yano $[4 ; 8]$ and it was proved that the conformal holonomy group of a space of type $\subseteq$ fixes an $(m-1)$-dimensional sphere or a point, according as the space is of type $\mathfrak{S}^{m-1}$ or $\mathfrak{S}^{\infty}$ (in the case where $m=0$, the fixed sphere should be considered as an imaginary one). Moreover, they also dealt with the converse problem.

The main purpose of this section is to establish

TheOREM 1. Let $g$ and $\bar{g}$ be two conformally equivalent Riemannian metrics which are complete and of type $\subseteq$.

(i) If $g$ is of type $\widetilde{S}^{m-1}$ and $m \geqq 1$, then $g$ and $\bar{g}$ coincide;

(ii) If $g$ is of type $\mathfrak{S}^{\infty}$, then the associated function of $\bar{g}$ with respect to $g$ is constant.

In the above theorem, let $f$ be an arbitrary element of $C(g)$. Then the Riemannian metric $f^{*} g$ is clearly complete and of type $\subseteq$. Now, by taking $\bar{g}=f^{*} g$, we have

THEOREM 2. Let $g$ be a complete Riemannian metric.

(i) If $g$ is of type $\mathfrak{S}^{m-1}$ and $m \geqq 1$, then $C(g)$ and $I(g)$ coincide;

(ii) If $g$ is of type $\mathfrak{S}^{\infty}$, then $C(g)$ is homothetic.

Proof of Theorem 1. The proof is divided into three steps.

I. In the following, by a projection of $F_{n}$, we mean an endomorphism $H$ of $F_{n}$ into itself satisfying (i) $H^{2}=H$ and (ii) $\phi\left(H \xi, \xi^{\prime}\right)=\phi\left(\xi, H \xi^{\prime}\right)$ for all $\xi$, $\xi^{\prime} \in F_{n}$. We shall say that a linear mapping $J$ of $F_{n}$ into $F_{n}^{*}$ is of type $\mathfrak{S}^{m-1}$, if there exists a projection $H^{\prime}$ of $F_{n}$ which is of rank $m$ and if it is expressed as 


$$
J=\frac{1}{2} \phi \circ H^{\prime}-\frac{1}{2} \phi \circ H^{\prime \prime},
$$

where $H^{\prime \prime}$ denotes the endomorphism of $F_{n}$ into itself defined by $H^{\prime \prime} \xi=\xi-H^{\prime} \xi$ for all $\xi \in F_{n}$.

Fix a linear mapping $J$ of $F_{n}$ into $F_{n}^{*}$ which is of type $\subseteq^{m-1}(0 \leqq m \leqq n)$ and consider the decomposition of $F_{n}$ associated with the projection $H^{\prime}$ :

$$
F_{n}=V^{\prime}+V^{\prime \prime}
$$

where $V^{\prime}=H^{\prime} F_{n}$ and $V^{\prime \prime}=H^{\prime \prime} F_{n}$. We denote by $\mathfrak{f}(J)$ the subalgebra of $\mathfrak{m}(n)$ generated by all the elements $\xi+J(\xi)$, where $\xi$ runs over $F_{n}$, and denote by $K(J)$ the subgroup of $M(n)$ generated by $\mathfrak{f}(J)$. By the stereographic projection $\xi \rightarrow \exp \xi \cdot o$, we define an $(m-1)$-dimensional sphere $\Phi_{J}$ of $M_{n}$ as follows:

$$
\Phi_{J}=\left\{\exp \xi^{\prime} \cdot o \mid \phi\left(\xi^{\prime}, \xi^{\prime}\right)=4, \xi^{\prime} \in V^{\prime}\right\} .
$$

Denote by $K_{J}$ the connected component of $M_{n}-\Phi_{J}$ which contains $\boldsymbol{o}$.

Then we have

Lemma 4. Let $J$ be a linear mapping of $F_{n}$ into $F_{n}^{*}$ which is of type $\mathfrak{S}^{m-1}$.

(i) Every element of $K(J)$ fixes $K_{J}$;

(ii) Every element $p$ of $K_{J}$ can be written as

$$
p=\exp (\xi+J(\xi)) \text { o for some } \xi \in F_{n} .
$$

Proof. Denote by $\mathfrak{l}^{\prime}$ (resp. $\left.\mathfrak{l}^{\prime \prime}\right)$ the subspace of $\mathfrak{f}(J)$ composed of all the elements $\xi^{\prime}+\phi\left(\xi^{\prime}\right) / 2$ where $\xi^{\prime} \in V^{\prime}$ (resp. $\xi^{\prime \prime}-\phi\left(\xi^{\prime \prime}\right) / 2$ where $\left.\xi^{\prime \prime} \in V^{\prime \prime}\right)$ and consider the subspace $\mathfrak{g}^{\prime}$ (resp. $\mathfrak{g}^{\prime \prime}$ ) of $\mathfrak{m}(n)$ as follows:

$$
\left.\mathfrak{g}^{\prime}=\mathfrak{l}^{\prime}+\mathfrak{o}(m) \text { (resp. } \mathfrak{g}^{\prime \prime}=\mathfrak{l}^{\prime \prime}+\mathfrak{o}(n-m)\right),
$$

where $\mathfrak{D}(m)$ (resp. $\mathfrak{D}(n-m)$ ) denotes the subalgebra of $\mathfrak{D}(n)$ which operates trivially on $V^{\prime \prime}$ (resp. $\left.V^{\prime}\right)$. An easy calculation shows that $\left[\mathfrak{l}^{\prime}, \mathfrak{l}^{\prime}\right]=\mathfrak{o}(m)$, $\left[\mathfrak{l}^{\prime}, \mathfrak{o}(m)\right]=\mathfrak{l}^{\prime},\left[\mathfrak{l}^{\prime \prime}, \mathfrak{l}^{\prime \prime}\right]=\mathfrak{o}(n-m),\left[\mathfrak{l}^{\prime \prime}, \mathfrak{o}(n-m)\right]=\mathfrak{l}^{\prime \prime}$ and $\left[\mathfrak{g}^{\prime}, \mathfrak{g}^{\prime \prime}\right]=0$. It follows that $\mathfrak{g}^{\prime}$ and $\mathfrak{g}^{\prime \prime}$ are subalgebras of $\mathfrak{m}(n)$ and that $\mathfrak{f}(J)$ is expressed as a direct sum of $\mathfrak{g}^{\prime}$ and $\mathfrak{g}^{\prime \prime}$ :

$$
\mathfrak{f}(J)=\mathfrak{g}^{\prime}+\mathfrak{g}^{\prime \prime} .
$$

In order to prove (i), it is sufficient to show that the elements of the form $\exp A$ where $A \in \mathrm{g}^{\prime}$ or $\mathrm{g}^{\prime \prime}$ fix $\Phi_{J}$. Let $p$ be an arbitrary point of $\Phi_{J}$. Then we see from (1.8) that it is expressed as $p=\omega\left(\xi_{0}+x^{\prime}+2 \xi_{\infty}\right)$, where $x^{\prime} \in V^{\prime}$. If $A \in \mathrm{g}^{\prime \prime}$, we have $\psi(A)\left(\xi_{0}+x^{\prime}+2 \xi_{\infty}\right)=0$, from which it follows that $\exp A \cdot p$ $=p$. Now assume that $A \in \mathfrak{g}^{\prime}$. We have $\psi(A)\left(\xi_{0}-2 \xi_{\infty}\right)=0$. If we set $\exp \psi(A)$ $\cdot\left(\xi_{0}+x^{\prime}+2 \xi_{\infty}\right)=y_{0} \xi_{0}+y^{\prime}+y_{\infty} \xi_{\infty}$, then we have $\phi\left(y_{0} \xi_{0}+y^{\prime}+y_{\infty} \xi_{\infty}, \xi_{0}-2 \xi_{\infty}\right)=0$, from which it follows that $4 y_{0}^{2}=\phi\left(y^{\prime}, y^{\prime}\right)$. We have

$$
\exp A \cdot p=\omega\left(y_{0} \xi_{0}+y^{\prime}+y_{\infty} \xi_{\infty}\right)=\exp \left(\left(1 / y_{0}\right) y^{\prime}\right) \cdot o\left(\in \Phi_{J}\right) \text {. }
$$


Now we shall prove (ii). For this purpose, we define four subsets $M_{m}$, $M_{n-m}, S_{m}^{0}$ and $S_{m}^{\infty}$ of $M_{n}$ as follows: $M_{m}=\left\{\exp \xi^{\prime} \cdot o \mid \xi^{\prime} \in V^{\prime}\right\} \cup\{\infty\}, M_{n-m}$ $=\left\{\exp \xi^{\prime \prime} \cdot o \mid \xi^{\prime \prime} \in V^{\prime \prime}\right\} \cup\{\infty\}, S_{m}^{0}=\left\{\exp \xi^{\prime} \cdot o \mid \phi\left(\xi^{\prime}, \xi^{\prime}\right)<4\right\}$ and $S_{m}^{\infty}=\{\exp$ $\left.\xi^{\prime} \cdot o \mid \phi\left(\xi^{\prime}, \xi^{\prime}\right)>4\right\} \cup\{\infty\}=\left\{\exp \phi\left(\xi^{\prime}\right) \cdot o \mid \phi\left(\xi^{\prime}, \xi^{\prime}\right)<1\right\}$. Then we have easily

$$
K_{J}=M_{n}-\Phi_{J}=M_{n-m} \cup\left\{M_{n}-\left(M_{m} \cup M_{n-m}\right)\right\} \cup S_{m}^{0} \cup S_{m}^{\infty} .
$$

Since we have, for each $\xi \in F_{n}$,

$$
\exp (\xi+J(\xi))=\exp \left(\xi^{\prime}+\phi\left(\xi^{\prime}\right) / 2\right) \exp \left(\xi^{\prime \prime}-\phi\left(\xi^{\prime \prime}\right) / 2\right),
$$

where $\xi^{\prime}=H^{\prime} \xi$ and $\xi^{\prime \prime}=H^{\prime \prime} \xi$, the statement of (ii) follows from the verification of the following four cases:

(a) For each $p \in M_{n-m}$, there is a $\xi^{\prime \prime} \in V^{\prime \prime}$ such that

$$
p=\exp \left(\xi^{\prime \prime}-\phi\left(\xi^{\prime \prime}\right) / 2\right) \cdot o \text {; }
$$

(b) For each $p \in M_{n}-\left(M_{m} \cup M_{n-m}\right)$, there are a $\xi^{\prime} \in V^{\prime}$ and a $q \in M_{n-m}$ such that $p=\exp \left(\xi^{\prime}+\phi\left(\xi^{\prime}\right) / 2\right) \cdot q$;

(c) For each $p \in S_{m}^{0}$, there is a $\xi^{\prime} \in V^{\prime}$ such that $p=\exp \left(\xi^{\prime}+\phi\left(\xi^{\prime}\right) / 2\right) \cdot o$;

(d) For each $p \in S_{m}^{\infty}$, there is a $\xi^{\prime} \in V^{\prime}$ such that $p=\exp \left(\xi^{\prime}+\phi\left(\xi^{\prime}\right) / 2\right) \cdot \infty$.

We write down the proof for case (b) only, since the other cases can be treated similarly by using Lemma 5 . Let $p$ be an arbitrary point of $M_{n}-\left(M_{m} \cup M_{n-m}\right)$. We see that $p$ is expressed as $p=\exp x \cdot o$, where $x \in F_{n}$. If we set $x^{\prime}=H^{\prime} x$ and $x^{\prime \prime}=H^{\prime \prime} x$, then we have $x^{\prime} \neq 0$ and $x^{\prime \prime} \neq 0$. Now define an element $\xi^{\prime}$ of $V^{\prime}$ and an element $y^{\prime \prime}$ of $V^{\prime \prime}$ as follows: $\xi^{\prime}=\left(\theta /\left(\phi\left(x^{\prime}, x^{\prime}\right)\right)^{1 / 2}\right) x^{\prime}$ and $y^{\prime \prime}=\left(\beta /\left(\phi\left(x^{\prime \prime}, x^{\prime \prime}\right)\right)^{1 / 2}\right) x^{\prime \prime}$, where $\beta$ and $\theta$ are respectively given by

$$
\beta=\frac{\phi(x, x)-4+\left((\phi(x, x)-4)^{2}+16 \phi\left(x^{\prime \prime}, x^{\prime \prime}\right)\right)^{1 / 2}}{2\left(\phi\left(x^{\prime \prime}, x^{\prime \prime}\right)\right)^{1 / 2}}
$$

and

$$
\sin \frac{\theta}{2}=\left(\frac{4\left(\beta-\left(\phi\left(x^{\prime \prime}, x^{\prime \prime}\right)\right)^{1 / 2}\right)}{\left(\phi\left(x^{\prime \prime}, x^{\prime \prime}\right)\right)^{1 / 2}\left(4+\beta^{2}\right)}\right)^{1 / 2} .
$$

Then, we have $p=\exp x \cdot o=\exp \left(\xi^{\prime}+2^{-1} \phi\left(\xi^{\prime}\right)\right) \cdot \exp y^{\prime \prime} \cdot o$. Indeed, if we set

$$
\rho=1+(\sinh \theta / 2)^{2}+4^{-1}(\sinh \theta / 2)^{2} \phi\left(y^{\prime \prime}, y^{\prime \prime}\right),
$$

then we have

$$
\rho x=(\sinh \theta / \theta)\left(1+4^{-1} \phi\left(y^{\prime \prime}, y^{\prime \prime}\right)\right) \xi^{\prime}+y^{\prime \prime} .
$$

Therefore, by Lemma 5 , we have

$$
\exp A^{\prime}\left(\xi_{0}+y^{\prime \prime}+\frac{\phi\left(y^{\prime \prime}, y^{\prime \prime}\right)}{2} \xi_{\infty}\right)=\rho\left(\xi_{0}+x+\frac{\phi(x, x)}{2} \xi_{\infty}\right)
$$

where $A^{\prime}=\psi\left(\xi^{\prime}+\phi\left(\xi^{\prime}\right) / 2\right)$. But, in view of (1.8), this is nothing but the de- 
sired formula. Since $q=\exp y^{\prime \prime} \cdot o \in M_{n-m}$, we have completed the proof of b). q.e.d.

Lemma 5.

(i) For each $\xi \in F_{n}$, set $A=\psi(\xi+\phi(\xi) / 2)$. Then, we have

$$
\exp A=1+(\sinh \theta / \theta) A+\left(\cosh \theta-1 / \theta^{2}\right) A^{2},
$$

where $\theta=(\phi(\xi, \xi))^{1 / 2}$

(ii) For each $\xi \in F_{n}$, set $A=\psi(\xi-\phi(\xi) / 2)$. Then, we have $\exp A=1+$ $(\sin \theta / \theta) A-\left((\cos \theta-1) / \theta^{2}\right) A^{2}$, where $\theta=(\phi(\xi, \xi))^{1 / 2}$.

We shall use

Lemma 6. Let $J$ and $\bar{J}$ be two linear mappings of $F_{n}$ into $F^{*}$ which are both of type $\varsigma^{m-1}$, where $m \geqq 1$. We assume that there exist an element $\rho$ of $R_{+}$and an element $E$ of $F_{n}^{*}$ such

$$
\rho \cdot \exp E \Phi_{J}=\Phi \bar{J} .
$$

Then, we have $\rho=1$ and $E=0$.

Proof. Let $H^{\prime}$ and $\bar{H}^{\prime}$ be the projections of $F_{n}$ defining $J$ and $\bar{J}$ respectively. If we set $V^{\prime}=H^{\prime} F_{n}$ and $\bar{V}^{\prime}=\bar{H}^{\prime} F_{n}$, then, in view of the definition of $\Phi_{J}$ and $\Phi_{\bar{J}}$, we see that there is a mapping $\xi \rightarrow \eta$ of $\left\{\xi \in V^{\prime} \mid \phi(\xi, \xi)=4\right\}$ onto $\left\{\eta \in \bar{V}^{\prime} \mid \phi(\eta, \eta)=4\right\}$ such that

$$
\rho \cdot \exp E \cdot \exp \xi \cdot o=\exp \eta \cdot o .
$$

But, by (1.8), we see that this is equivalent to the following two formulae:

$$
\begin{aligned}
& \rho^{2}=1+\langle\xi, E\rangle+\left\langle\phi^{-1} E, E\right\rangle ; \\
& \rho \eta=\xi+2 \phi^{-1} E .
\end{aligned}
$$

Observing that $-\xi \in\left\{\xi \in V^{\prime} \mid \phi(\xi, \xi)=4\right\}$, it follows from formula (i) that $\rho^{2}=1+\left\langle\phi^{-1} E, E\right\rangle$. Moreover, from formulae (i) and (ii), we have $\rho^{2}$ $=1+\rho\langle\eta, E\rangle-\left\langle\phi^{-1} E, E\right\rangle$; as above, it follows that $\rho^{2}=1-\left\langle\phi^{-1} E, E\right\rangle$. Therefore, we have $\rho=1$ and $E=0$. q.e.d.

II. Let $g$ be a Riemannian metric on a manifold $M$. Let $P_{S}$ be the corresponding conformal $S(n)$-bundle and let $P^{\prime}$ together with $l$ and $\alpha$ be the conformal $M^{\prime}(n)$-bundle associated with $P_{S}$. Let $P_{0}$ be the orthogonal bundle corresponding to $g$ and let $h$ be the corresponding injection of $P_{0}$ into $P^{\prime}$. Under the assumption that $g$ is of type $\mathfrak{S}$, we define, at each point $p$ of $M$, two subsets $\Phi_{o}(p)$ and $K_{o}(p)$ of the tangent Möbius space $M_{n}(p)$ at $p$ as follows:

The case where $\mathrm{g}$ is of type $\mathfrak{S}^{m-1}$. In this case, the Ricci tensor field $S$ of $g$ is expressed as $S=(m-1) g \circ H^{\prime}-(n-m-1) g \circ H^{\prime \prime}$. For each $x \in P_{0}$, we denote by $H_{x}^{\prime}$ the endomorphism of $F_{n}$ into itself which is defined by $H_{x}^{\prime}$ $=x^{-1} \cdot H^{\prime} \cdot x$. Let $B_{0}$ be the Riemannian connection in $P_{0}$. If we observe that 
$S\left(x \cdot \xi, x \cdot \xi^{\prime}\right)=S_{x}\left(\xi, \xi^{\prime}\right)$ and $g\left(x \cdot \xi, x \cdot \xi^{\prime}\right)=\phi\left(\xi, \xi^{\prime}\right)$, an easy calculation shows that the linear mapping $J_{x}$ which is defined by formula (2.2) starting with the Riemannian connection $B_{0}$ is given by the formula $J_{x}=2^{-1} \phi \circ H_{x}^{\prime}$ $-2^{-1} \phi \circ H_{x}^{\prime \prime}$, where $H_{x}^{\prime \prime} \xi=\xi-H_{x}^{\prime} \xi$ for all $\xi \in F_{n}$. This means that $J_{x}$ is of type $\widetilde{S}^{m-1}$. Therefore, the argument in II applies to $J_{x}$. We now define $\Phi_{\boldsymbol{g}}(p)$ and $K_{0}(p)$ as follows:

$$
\Phi_{\sigma}(p)=h(x) \cdot \Phi_{J_{x}} ; \quad K_{g}(p)=h(x) \cdot K_{J_{x}},
$$

where $x$ is an element of $P_{0}$ such that $\pi_{0}(x)=p$. The definition does not depend on the choice of $x \in P_{0}$ such that $\pi_{0}(x)=p$. Indeed, if we set $V_{x}^{\prime}=H_{x}^{\prime} F_{n}$, then we have $V_{x \cdot \sigma}^{\prime}=\sigma^{-1} V_{x}^{\prime}$ for each $x \in P_{0}$ and $\sigma \in O(n)$. It follows immediately from the definition of $\Phi_{J}$ and $K_{J}$ that $\Phi_{J_{x \cdot \sigma}}=\sigma^{-1} \Phi_{J_{x}}$ and $K_{J_{x \cdot \sigma}}=\sigma^{-1} K_{J_{x}}$.

The case where $g$ is of type $\mathfrak{S}^{\infty}$. In this case, we define $\Phi_{g}(p)$ and $K_{o}(p)$ as follows:

$$
\Phi_{g}(p)=h(x) \cdot \infty ; \quad K_{\vartheta}(p)=h(x) \cdot E_{n},
$$

where $x$ is an element of $P_{0}$ such that $\pi_{0}(x)=p$ and where $E_{n}=M_{n}-\{\infty\}$, as was defined in (1.8). The definition is independent of the choice of $x \in P_{0}$ such that $\pi_{0}(x)=p$, because $O(n)$ fixes $\infty$.

In each case, $K_{g}(p)$ is an open submanifold of $M_{n}(p)$ which contains the origin $p^{*}$, and $\Phi_{g}(p)$ is the boundary of $K_{g}(p)$.

Lemma 7. Assume that $\mathrm{g}$ is a complete Riemannian metric of type $\mathfrak{S}$.

(i) At each point $p$ of $M$, every curve through $p$ in $M$ is developed into the part $K_{\theta}(p)$ of the tangent Möbius space $M_{n}(p)$ at $p$ with respect to the normal conformal connection associated with $P_{S}$.

(ii) For each point $p$ of $M$ and each point $q^{*}$ of $K_{\theta}(p)$, there exists a curve in $K_{o}(p)$ which joins $p^{*}$ (the origin of $M_{n}(p)$ ) and $q^{*}$ and which admits the development into the base space.

Proof. Fix a point $x$ of $P_{0}$ such that $\pi_{0}(x)=p$ and identify $F_{n}$ (resp. $M_{n}$ ) with the tangent Euclidean space $M_{p}$ (resp. the tangent Möbius space $\left.M_{n}(p)\right)$ at $p$ by $x$ (resp. $\left.h(x)\right)$. In the following, we use the notation in Proposition 6.

The case where $g$ is of type $\varsigma^{m-1}$. Let $u(t)$ be an arbitrary curve through $p$ in $M$. We know from Proposition 6 that the conformal development $u^{*}(t)$ of $u(t)$ at $p$ is given by $u^{*}(t)=a(t) o$, where $a(t)$ is the curve in $M(n)$ which is uniquely determined by the differential equation $a(t)^{-1} a^{\prime}(t)=v^{\prime}(t)+J_{x(t)}\left(v^{\prime}(t)\right)$ with the initial condition $a(0)=e$. Since the Ricci tensor field $S$ of $g$ is parallel, we see that $S_{x(t)}$ is constant for any horizontal curve in $P_{0}$ (with respect to the Riemannian connection in $P_{0}$ ). It follows from formula (2.2) that $J_{x(t)}$ is constant, hence, $v^{\prime}(t)+J_{x(t)}\left(v^{\prime}(t)\right)=v^{\prime}(t)+J_{x}\left(v^{\prime}(t)\right)$ is contained in $\mathfrak{t}\left(J_{x}\right)$. It follows that $a(t)$ is a curve in $K\left(J_{x}\right)$, because $K\left(J_{x}\right)$ is the subgroup of $M(n)$ generated by $\mathfrak{f}\left(J_{x}\right)$. Therefore, by (i) of Lemma 4 , we see that 
$a(t) o$ is a curve in $K_{J_{x}}$, which proves (i) (note the $K_{J_{x}}$ is identified with $K_{0}(p)$ by $\left.h(x)\right)$.

Now we shall prove (ii). Let $q^{*}$ be an arbitrary element of $K_{J_{x}}$. We know from (ii) of Lemma 4 that there is an element $\xi$ of $F_{n}$ such that $q^{*}$ $=\exp (\xi+J(\xi))$. Now consider the geodesic $u(t)$ of $g$ such that $u^{\prime}(0)=\xi$ (we identify $\xi$ with a vector in $M_{p}$ by $x$ ). Since the metric $g$ is complete, the geodesic $u(t)$ is defined for any real number $t$. We show that the conformal development $u^{*}(t)$ of $u(t)$ at $p$ joins $p^{*}$ and $q^{*} . u(t)$ being a geodesic such that $u^{\prime}(0)=\xi$, the Euclidean development $v(t)$ of $u(t)$ at $p$ is given by $v(t)=t \xi$. Hence, by Proposition 6 , the conformal development $u^{*}(t)$ of $u(t)$ at $p$ is given by $u^{*}(t)=a(t) o$, where $a(t)^{-1} a^{\prime}(t)=\xi+J_{x(t)}(\xi)=\xi+J_{x}(\xi)$, that is, $a(t)$ $=\exp t\left(\xi+J_{x}(\xi)\right)$. We have $u^{*}(1)=a(1) o=q^{*}$, which proves our assertion, we have thereby proved (ii).

The case where $g$ is of type $\subseteq^{\infty}$. The proof is similar to the case where $g$ is of type $\widetilde{S}^{m-1}$. We remark the following points. The linear mapping $J_{x}$ of $F_{n}$ into $F_{n}^{*}$ which is defined by formula $(2.2)$ reduces to zero. The stereographic projection $\xi \rightarrow \exp \xi \cdot o$ gives a one-to-one correspondence of $F_{n}$ with $E_{n}$, and $E_{n}$ is identified with $K_{\theta}(p)$ by $h(x)$. We have thus completed the proof of Lemma 7.

Remark. A theorem of K. Yano and S. Sasaki [4;8] states that the assignment $p \rightarrow \Phi_{g}(p)$ is parallel with respect to the normal conformal connection, which is almost evident by Lemma 7 , if we remark that $\Phi_{g}(p)$ is the boundary of $K_{\imath}(p)$.

III. Now consider a second Riemannian metric $\bar{g}$ on $M$ which is complete and of type $\subseteq$ and which is conformally equivalent to $g$. Let $\bar{P}_{0}$ and $\bar{h}$ be the corresponding orthogonal bundle and injection of $\bar{P}_{0}$ into $P^{\prime}$. At each point $p$ of $M$, we define $\Phi_{\bar{\theta}}(p)$ and $K_{\bar{\theta}}(p)$ as in II starting with $\bar{g}$.

LEMMA 8. $\Phi_{g}(p)=\Phi_{\bar{g}}(p)$ at each point $p$ of $M$.

Proof. $\Phi_{g}(p)$ (resp. $\Phi_{\bar{g}}(p)$ ) is the boundary of $K_{g}(p)$ (resp. $K_{\bar{\theta}}^{-}(p)$ ). Therefore, it is sufficient to prove that $K_{g}(p)=K_{\bar{\theta}}^{-}(p)$. Let $q^{*}$ be an arbitrary point of $K_{g}(p)$. We know from (ii) of Lemma 7 that there is a curve $u^{*}(t)$ in $K_{\theta}(p)$ joining $p^{*}$ and $q^{*}$ which admits the development $u(t)$ into the base space. Now, by applying (i) of Lemma 7 to $\bar{g}$, we see that $u^{*}(t)$ is a curve in $K_{\bar{g}}(p)$ and, in particular, $q^{*}$ is contained in $K_{g}(p)$. Thereby we have proved that $K_{g}(p) \subset K_{\bar{g}}(p)$. In the same way, we have $K_{\bar{g}}(p) \subset K_{g}(p)$, whence $K_{\theta}(p)$ $=K_{g}(p)$. q.e.d.

By using Lemma 8, we now prove Theorem 1. To do this, we need several formulae concerning the relation between $P_{0}$ and $\bar{P}_{0}$. Let $s$ (resp. $\bar{s}$ ) be the homomorphism of $P_{S}$ onto $P_{0}$ (resp. $\bar{P}_{0}$ ) defined in $\$ 2$ and let $\lambda$ be the associated function of $\bar{g}$ with respect to $g$. By Proposition 3 , applied to $\bar{P}_{0}$, there exist mappings $\bar{\rho}$ and $\bar{E}$ of $P^{\prime}$ into $R_{+}$and $F_{n}^{*}$ respectively such that 
$z=\bar{h} \circ \bar{s} \circ l(z) \cdot \bar{\rho}(z) \cdot \exp \bar{E}(z)$ and $\alpha=\bar{\rho}^{-1} d \bar{\rho}+[\theta, \bar{E}]_{R}$. If we set $\rho=\bar{\rho} \circ h$ and $E=\bar{E} \circ h$, then it follows that

$$
\begin{aligned}
h(x) & =\bar{h} \circ \bar{s}(x) \cdot \rho(x) \cdot \exp E(x) & & \text { for all } x \in P_{0} ; \\
\rho^{-1} d \rho+\left[\theta^{\prime}, E\right]_{R} & =0, & & \text { where } \theta^{\prime}=h^{*} \theta ; \\
\rho(x) & =\lambda \circ \pi_{S}(x) . & &
\end{aligned}
$$

(6.2) follows from the fact that $h^{*} \alpha=0$ and (6.3) is obtained by applying $l$ to formula (6.1) and taking account of formula (2.1).

(i) The case where $g$ is of type $\mathfrak{S}^{m-1}(m \geqq 1)$. By Lemma 8 , we see that $\bar{g}$ is also of type $\varsigma^{m-1}$. Fix a point $x$ of $P_{0}$ and set $y=\bar{s}(x)$ and $p=\pi_{0}(x)$. We have

$$
\Phi_{g}(p)=h(x) \cdot \Phi_{J_{x}} \quad \text { and } \quad \Phi_{\bar{g}}^{-}(p)=\bar{h}(y) \cdot \Phi_{J_{y}}
$$

where $\bar{J}_{y}$ denotes the linear mapping of $F_{n}$ into $F_{n}^{*}$ defined by formula (2.2) starting with $\bar{g}$. It follows from Lemma 8 and formula (6.1) that

$$
\rho(x) \cdot \exp E(x) \Phi_{J_{x}}=\Phi_{\bar{J}_{y}} .
$$

Therefore, by Lemma 6 , we have $\rho(x)=1$ and hence, by formula (6.3), $\lambda(p)=1$. $p$ being arbitrary, we have $\lambda=1$, whence $g=\bar{g}$.

(ii) The case where $g$ is of type $\widetilde{S}^{\infty}$. It follows from Lemma 8 that $\bar{g}$ is also of type $\varsigma^{\infty}$. Fix a point $x$ of $P_{0}$ and set $y=\bar{s}(x)$ and $p=\pi_{0}(x)$. We have

$$
\Phi_{\theta}(p)=h(x) \cdot \infty \quad \text { and } \quad \Phi_{\bar{g}}(p)=\bar{h}(y) \cdot \infty .
$$

It follows from Lemma 8 and formula (6.1) that

$$
\rho(x) \cdot \exp E(x) \cdot \infty=\infty \text {. }
$$

But, by (1.8), we have

$$
\rho(x) \cdot \exp E(x) \cdot \infty=\omega\left(1 / 2 \rho(x)\left\langle\phi^{-1} E(x), E(x)\right\rangle \xi_{0}+\phi^{-1} E(x)+\rho(x) \xi_{\infty}\right) ;
$$

furthermore, we have $\infty=\omega\left(\xi_{\infty}\right)$. It follows immediately that $E(x)=0$. Now, by formula (6.2), we see that $\rho$ is constant and hence, by formula (6.3) that $\lambda$ is constant, which proves our assertion. Thus we have completed the proof of Theorem 1.

\section{BIBLIOGRAPHY}

1. E. Cartan, Les espaces à connexion conforme, Ann. Soc. Polon. Math. vol. 2 (1923) pp. 171-221.

2. C. Ehresmann, Les connexions infinitésimales dans un espace fibré differentiable, Colloque de Topologie, Bruxelles, 1950, pp. 29-55. nese).

3. S. Sasaki, Geometry of the conformal connection, Kawade Shobô, Tôkyo, 1948 (in Japa- 
4. - On the spaces with normal conformal connections whose groups of holonomy fix a point or a hypersphere, I, II, III, Jap. J. Math. vol. 34 (1942) pp. 623-633; vol. 35 (1943) pp. 791-795.

5. N. Tanaka, Projective connections and projective transformations, Nagoya Math. J. (1957) pp. 1-24.

6. K. Nomizu, Lie groups $a_{i}^{\prime}{ }^{\prime}$ differential geometry, Publications Math. Soc. Japan, no. 2, 1956.

7. K. Yano, Sur la theorie des espaces a connexion conforme, J. Fac. Sci. Imp. Univ. Tôkyo. Sect. 1, vol. 4 (1939) pp. 1-59.

8. K. Yano and S. Sasaki, Sur les espaces d connexion conforme normale dont les groupes d'holonomie fixent une sphère d un nombre quelconque de dimensions I, Proc. Imp. Acad. Japan vol. 20 (1944) pp. 525-535.

Nagoya UNIVERSITY,

NAGOYA, JAPAN 